Relations industrielles

Industrial Relations

\title{
Vers une union des familles
}

Volume 6, numéro 3, juin 1951

URI : https://id.erudit.org/iderudit/1023216ar

DOI : https://doi.org/10.7202/1023216ar

Aller au sommaire du numéro

Éditeur(s)

Département des relations industrielles de l’Université Laval

ISSN

0034-379X (imprimé)

1703-8138 (numérique)

Découvrir la revue

Citer cet article

(1951). Vers une union des familles. Relations industrielles / Industrial Relations, 6(3), 89-91. https://doi.org/10.7202/1023216ar

Tous droits réservés (C Département des relations industrielles de l’Université Laval, 1951
Ce document est protégé par la loi sur le droit d'auteur. L'utilisation des services d'Érudit (y compris la reproduction) est assujettie à sa politique d'utilisation que vous pouvez consulter en ligne.

https://apropos.erudit.org/fr/usagers/politique-dutilisation/ 
- risque d'affamer des milliers d'hommes, toute l'immense multitude des malheureux .... * Ce problème du chômage périodique, dit M. Bayart, c'est le problème crucial du capitalisme, et plus exactement d'un régime économique fondé sur le développement dynamique du progrès technique qui, par le mouvement qu'il imprime à l'activité économique, engendre nécessairement, dans sa marche en avant, des variations, les fluctuations de la conjoncture et des crises, cycliques ou non ». Voici le résumé de la Pensée pontificale dans ce discours du 3 juin, tel que présenté par M. Bayart :

Le rejet des \& mécanismes d'organisations sociales en contradiction avec l'ordre établi par Dieu », c'està-dire de la planification intégrale du système soviétique;

le rejet d'un retour pur et simple a un libéralisme condamné par l'Eglise et dépassé par les faits, qui se fierait, pour rétablir une économie normale, au seul mécanisme des lois du marché ;

la mise en garde contre une déformation de la sécurité sociale par une conception socialisante qui \& chargerait un Etat-Providence du soin de procurer à chacun de ses ressortissants, et pour toutes les circonstances de la vie, le droit à des exigences au bout du compte irréalisables »;

la mise en garde contre certaines conceptions de plein emploi réalisé par des formalismes trop artificiels; la nécessité primordiale, pour réaliser cette politique du plein emploi, et la première des sécurités sociales: celle du travail, d'organiser, à la source même de l'économie, et en évitant les excès d'un productivisme sans frein, une \& productivité saine et normale, sagement mesurée aux besoins et à la dignité de l'homme , et ordonnée autour de cette unité organique, constitutive de la' Société, qu'est la Famille ;

le maintien, à la base de l'économie, de l'entreprise privée, avec toutes les amodiations correspondant à son caractère social, mais en maintenant néanmoins son caractère privé et en laissant au chef d'entreprise sa responsabilité personnelle et la maîtrise de ses décisions économiques, et en sauvegardant autant que possible, contre le double danger du capitalisme anonyme et de la socialisation, cette portion la plus saine de l'économie que sont les exploitations individuelles ou familiales ;

l'affirmation, une fois de plus réitérée, de la nécessité primordiale d'une organisation professionnelle.

Tel est le sens de l'orientation nouvelle que le Très Saint Père indique à l'économie mondiale. Il invite maintenant théoriciens et techniciens « à concentrer leur attention et à faire converger leurs études . . . sur l'imminente et permanente menace du chômage, le plus imnortant, le plus urgent problème, celui qui pèse comme un cauchemar, précisément sur ces vieux pays d'industrie. . .»

\section{Vers une union des familles}

Les 23 et 24 avril dernier, le Département des relations industrielles de la Faculté des sciences sociales de Laval accueillait, dans la vaste salle de spectacles du Château Frontenac, près de cinq cents délégués venus de tous les coins de la province et des milieux les plus divers: patrons, ouvriers, directeurs de personnel, con- tremaîtres, officiers d'associations patronales et ouvrières, représentants de comités paritaires, aviseurs moraux, fonctionnaires du travail, représentants de groupements sociaux, etc.

Deux jours durant, les congressistes ont considéré, dans la sérénité qui convient et sous la direction éclairée de savants conférenciers et 
de comités formés d'experts praticiens, l'aspect théorique de la sécurité sociale en fonction de la famille ouvrière, ainsi que ses principales modalités d'application.

Les deux premières séances étaient réservées à l'étude des principes de la sécurité et particulièrement au plein-emploi en tant qu'élément fondamental de la sécurité de la famille ouvrière. Un grand intérêt et une attention soutenue se sont prolongés durant l'examen des mesures pratiques projetées ou déjà en voie de réalisation en notre pays.

Le conférencier invité à l'occasion du dîner de clôture du Congrès, le R. P. Gonzalve Poulin, o.f.m., directeur de l'Ecole de service social de Laval, a voulu pousser l'examen du problème au delà de la sécurité économique de la famille ouvrière. Après avoir analysé les deux facteurs de déséquilibre familial que sont l'urbanisation improvisée et le changement de la fonction sociale de la propriété privée, le conférencier a esquissé les voies possibles de solution dans une meilleure organisation du milieu industriel, du milieu social et du milieu familial. Quant aux conditions de la stabilité familiale, le conférencier les a résumées aux trois suivantes : l'humanisation du milieu de travail, la promotion de mesures de sécurité sociale et l'organisation de la famille sur le plan social-politique. Cette dernière condition présentée dans une perpective nouvelle mérite qu'on s'y arrête particulièrement et l'extrait suivant de cette magistrale causerie doit être porté à l'attention de nos lecteurs :

\& C'est peut-être sur le plan sociopolitique que l'enracinement de la famille ouvrière reste le plus ardu. Parce que l'organisation du capital et du travail n'influence la vie politique que dans le sens des intérêts économiques, il en résulte une démocratie de caractère individualiste et dont les lois apparemment sociales, comme les mesures de sécurité, s'a- dressent plutôt à l'individu qu'au groupe familial. Si la famille, par l'association, devenait une force sociale, elle ferait reconnaître ses droits politiques et sociaux, accentuerait le caractère communautaire de la soeiété et rendrait plus facile l'intégration pacifique de la famille ouvrière dans la communauté nationale.

Il en résulterait un lien social plus fort entre les divers groupes de la nation, et, chez les responsables de l'Etat, une prise de conscience de la réalité familiale. Il n'y a pas si long. temps que la famille formait le centre de la vie politique, économique et sociale !

Une union des familles assurerait une meilleure éducation des citoyens par un jeu plus normal des solidarités sociales et la diffusion des institutions d'entr'aide familiale telles que mutuelles, coopératives, services familiaux, etc. La moralité publique et les coutumes sociales en recevraient un contrôle éclairé. La famille ouvrière, alliée aux familles des autres classes sociales, n'aurait plus l'impression d'être mise au ban de la société moderne. Elle apporterait sa participation active au bien commun de la cité dans une atmosphère de confiance indispensable à son épanouissement et à sa promotion.

Si l'union des familles, en plus de telles fonctions sociales, assumait un rôle de représentation politique auprès des pouvoirs publics; si, par le truchement d'un conseil supérieur du bien-être des familles, elle conseillait l'Etat dans sa législation sociale, limitant les fonctions exécutrices de ce dernier qui s'immisce de plus en plus dans la vie privée des citoyens au détriment de ses responsabilités supplétives et régulatrices; si elle faisait accepter un code de la famille et corrigeait ainsi le caractère trop individualiste du droit moderne, peutètre verrait-on un mouvement de décentralisation et de déconcentration gagner toutes les sphères de la vie industrielle et sociale. La fonction 
organique que jouait la famille d'hier dans la civilisation rurale renaîtrait, mais sous des formes adaptées à la civilisation industrielle.

L'initiative de l'organisation des forces familiales revient évidemment à la famille elle-même; nous croyons toutefois que l'Etat, dans l'intérêt commun, devrait stimuler la naissance et le développement de cette organisation en lui assurant une place de représentation dans ses commissions et organismes publics. »

En concluant, l'éminent conférencier a rappelé avec à propos que « toute restauration économico-sociale de la famille ouvrière est vouée à l'insuccès à moins d'être accompagnée d'un renouveau de vie chrétienne. » "Si l'oeuvre de restauration, écrit l'épiscopat de la province de Québec, s'accomplit à la lumière des vérités éternelles de justice et de charité, les parties en présence demanderont à Dieu et à la religion la vue nette de leur fonction respective dans la société et de leur nécessaire collaboration au bien commun ainsi que la force surnaturelle de remplir leur: devoirs. Au lieu de chercher à maintenir les positions acquises ou viser à renverser simplement l'ordre établi, elles essaieront sincèrement de con- server ce qui est légitime et de remplacer ce qui ne mérite pas de vivre. Et ainsi la religion par ses enseignements sur l'homme, sa famille, ses loisirs et son travail, par la grâce indispensable à toute oeuvre de bien sera' la base d'une restauration chrétienne de la vie ouvrière. »

Ces assises, de l'aveu même des congressistes ont été une magnifique occasion d'échanges de vues et de contacts utiles. Mais il y a plus. A l'occasion du soixantième anniversaire de la publication de l'Encyclique Rerum Novarum, le Département des relations industrielles se devait de toucher de plus près le problème ouvrier, d'en examiner les causes profondes pour en découvrir à la lumière de la doctrine sociale de l'Eglise les solutions possibles. Il n'a pas voulu se dérober à la tâche, même au risque de se limiter à quelques points essentiels. En ce faisant, il a conscience d'avoir cherché à promouvoir dans son milieu et dans la mesure de ses moyens la paix et la justice sociales auxquelles tous aspirent ardemment.

N.B.-Les travaux présentés à l'occasion du sixième Congrès des relations industrielles seront réunis en un rapport qui sera mis en vente prochainement au Département des relations industrielles, 2 , rue de l'Université, Québec.

\section{SERVICE SOCIAL}

REVUE PROFESSIONNELLE DE SERVICE SOCIAL publiée par l'Ecole de service social affiliée à la Faculté des sciences sociales de l'Université Laval,

\section{VIENT DE PARAîTRE}

cette revue trimestrielle servira de véhicule à la pensée, à la culture propre, aux techniques et aux espoirs des travailleurs sociaux d'expression française en matière de service social;

cette revue d'information sociale contiendra une section consacrée aux articles de fonds, une section de documents, une section d'histoire, une section de nouvelles;

cette revue vous parviendra tous les trois mois moyennant contribution annuelle de deux dollars ou soixante cents pour chaque numéro.

\section{2, RUE DE L'UNIVERSITÉ}

QUÉBEC 